\title{
Impacto de Salud Oral sobre Calidad de Vida en Adultos Jóvenes de Clínicas Odontológicas Universitarias
}

\author{
Oral Health Impact on Life Quality in Young Adults at Dental University Clinics
}

\begin{abstract}
Shyrley Díaz-Cárdenas; Madera Anaya Meisser-Vidal'; Lesbia Rosa Tirado-Amador³; Natalia Fortich-Mesa ${ }^{4}$; Liliana Tapias-Torrado ${ }^{5}$ \& Farith Damián González-Martínez ${ }^{6}$
\end{abstract}

DÍAZ-CÁRDENAS, S.; MEISSER-VIDAL, M. A.; TIRADO-AMADOR, L. R.; FORTICH-MESA N.; TAPIAS-TORRADO, L. \& GONZÁLEZ-MARTíNEZ, F. D. Impacto de salud oral sobre calidad de vida en adultos jóvenes de clínicas odontológicas universitarias. Int. J. Odontostomat., 11(1):5-11, 2017.

RESUMEN: El objetivo de este estudio es asociar estado de salud oral y calidad de vida en adultos de clínicas odontológicas universitarias de Cartagena, Colombia. Material y Métodos: Estudio analítico, en 400 adultos jóvenes y maduros (20 a 59 años), que asistieron a las clínicas odontológicas de la Universidad de Cartagena y Corporación Universitaria Rafael Núñez, distribuidos equitativamente; se aplicó un instrumento validado para indagar variables sociodemográficas, el General Oral Health Assesment Index (GOHAl) para evaluar impacto calidad de vida relacionada con la salud oral (CVRSB) y examen oral para evaluar número de dientes, presencia de caries dental, placa bacteriana, restos radiculares, cálculo dental y obturaciones. Se analizaron datos a través de frecuencia y proporciones, significancia de relaciones entre variables $\left(\chi^{2}\right)$, estimación del riesgo $(\mathrm{OR})$ y modelo de regresión logística con un intervalo de confianza $95 \%$. Resultados. $66,7 \%$ de los participantes eran mujeres y se hallaban en adultez madura (54,7\%) edad promedio 44,6 años (DE=13,7). El $33,2 \%$ presentó un impacto negativo de CVRSB (IC $95 \%$ ). Se hallaron asociaciones entre bajo nivel de escolaridad $(\mathrm{OR}=2,4$; IC $95 \%=1,6-3,8 ; p=0,000)$, ausencia de servicios de salud $(O R=1,9 ;$ IC $95 \%=1,2-2,8 ; p=0,002)$, presencia de menos de 19 dientes $(O R=3,6 ;$ IC $95 \%=2,3-5,8 ; p=0,000)$, restos radiculares $(O R=6,5 ; I C \quad 95 \%=4,1-10 ; p=0,000)$ y cálculos (OR=5,3; IC $95 \%=3,3-8,4 ; p=0,000 ;)$ con el impacto negativo de CVRSB. En el modelo multivariado las variables que mejor explican el impacto negativo fueron: ausencia de servicios de salud, contar con menos de 19 dientes, presencia de restos radiculares y cálculos $(p<0,05)$. Conclusión. Adultos jóvenes y maduros presentan un impacto negativo de CVRSB al contar con menos de 19 dientes, presencia de restos radiculares y cálculos dentales y ausencia de servicios de salud.

PALABRAS CLAVE: salud oral, calidad de vida, auto percepción.

\section{INTRODUCCIÓN}

La calidad de vida ha sido definida por la Organización mundial de la salud (OMS) como: "Percepción que un individuo tiene de su lugar en la existencia, en el contexto de la cultura y de valores en los que vive y en relación con sus objetivos, expectativas, normas, inquietudes, los cuales están relacionados con la salud física, el estado psicológico, el grado de independencia, las relaciones sociales y las creencias religiosas" (WHOQOL Group, 1993; Hernandez et al.,
2006). Misrachi (2005), la define como: "Combinación de condiciones de vida y satisfacción personal ponderadas por escala de valores, aspiraciones y expectativas personales; además de considerar algunos aspectos de la vida de las personas, como el lugar donde viven, el modo de comportarse, su entorno en general, relaciones familiares y apoyos sociales, la satisfacción laboral, el estado funcional y disponibilidad económica; así como la salud general". En relación a

\footnotetext{
${ }^{1}$ Magíster en Salud Pública, Especialista en Salud Familiar y Gerencia en Salud, Odontóloga, Profesor Asistente, Facultad de Odontología, Universidad de Cartagena, Cartagena, Colombia.

${ }^{2}$ Magister en Epidemiología Clínica, Magister en Bioquímica, Odontólogo, Investigador Grupo GISPOUC, Facultad de Odontología, Universidad de Cartagena, Cartagena, Colombia.

${ }^{3}$ Odontóloga, Investigador Grupo GISPOUC, Facultad de Odontología, Universidad de Cartagena, Cartagena, Colombia.

${ }^{4}$ Magister en Epidemiología Clínica, Profesora Escuela de Odontología Corporación Universitaria Rafael Núñez, Cartagena, Colombia.

${ }^{5}$ Profesora Escuela de Odontología Corporación Universitaria Rafael Núñez Cartagena, Cartagena, Colombia.

${ }^{6}$ Magister en Salud Pública, Especialista en Investigación Social, Odontólogo, Profesor Asociado, Facultad de Odontología, Universidad de Cartagena, Cartagena, Colombia.
} 
la salud, la calidad de vida influye en factores que comprometen el bienestar biosicosocial como alimentación adecuada, medio ambiente favorable, estabilidad económica y acceso a servicios de salud (Montes et al., 2001; Meller et al., 2008).

La salud oral, parte integral y esencial para la salud en general puede también comprometer la calidad de vida de las personas (Gherunpong et al., 2004; Locker \& Allen, 2007; Díaz et al., 2012). Por ello es importante adquirir buenos hábitos de salud y en especial de salud oral desde edades tempranas con disminución de factores de riesgo en la adultez mayor que afecten la calidad de vida (Kushnir et al., 2004; Zini \& Sgan-Cohen, 2008) y generen altos costos en salud. La presencia o ausencia de algunas afecciones desde la adultez joven como enfermedad periodontal, caries dental, dientes perdidos y restaurados generan dolor, incapacidad para sonreír, deglutir, masticar, degustar y besar (comprometiendo bienestar sicosocial e influir en autoestima, expresión, comunicación y estética facial y hasta en la vida laboral originando ausentismo laboral y por ende baja producción económica (Bennadi \& Reddy, 2013); también estas afecciones orales pueden generar un impacto negativo en la salud, como mayor riesgo de diabetes descompensada, artritis, eventos trombóticos, partos pre término y otras enfermedades (Zini \& SganCohen) que podría complicarse hacia la adultez mayor. Muchos estudios han reportado estas asociaciones pero principalmente en adultos mayores, como Meller et al., Montes et al., Concha et al. (2007) y Díaz et al., pero existen pocos estudios locales que muestren asociación entre salud oral y la calidad de vida de adultos jóvenes y maduros; por ello el objetivo de este estudio fue asociar el estado de salud oral y la calidad de vida en adultos jóvenes y maduros atendidos en clínicas odontológicas universitarias de Cartagena de Indias, Colombia.

\section{MATERIAL Y MÉTODO}

Estudio analítico transversal, en 400 pacientes adultos atendidos en las Clínicas Odontológicas de la Universidad de Cartagena y la Corporación Universitaria Rafael Núñez, segundo periodo 2013 teniendo en cuenta adultos que aceptaron participar en el estudio a través de la firma del consentimiento informado y que no presentaban discapacidad mental y sensorial motora, teniendo en cuenta las dispo- siciones las disposiciones internacionales: Declaración de Helsinki (modificación de Edimburgo 2000) y normativa para Colombia (Resolución 8.430 de 1993, Ministerio de Salud).

La recolección de información se realizó en dos etapas: la primera consistió en la aplicación de un cuestionario previamente validado que indagaba características sociodemográficas y relacionadas con la salud oral: sexo, edad, estratificación social, nivel de escolaridad, estado civil, ocupación, religión, procedencia, seguridad social en salud, última visita al Odontólogo, motivo de consulta y tratamiento realizado. Se aplicó el General Oral Health Assesment Index (GOHAI) (Atchison \& Dolan, 1990), para evaluar los niveles de percepción de CVRSB; cuestionario validado de 12 preguntas que contempla tres dimensiones: función física, la cual tiene en cuenta la influencia en el comer, deglutir y hablar; función psicosocial, que indaga sobre la preocupación por su salud oral, insatisfacción con la apariencia, dificultad en el contacto social debido a problemas orales y por último función del dolor, mostrando la incomodidad reflejada en la cavidad oral y el uso de fármacos para aliviar el dolor. Las respuestas se codificaban en una escala de cinco puntos clasificadas como: $0=$ nunca, $1=$ rara vez, $2=$ a veces, $3=$ a menudo, $4=$ muy a menudo y $5=$ siempre. La segunda etapa consistió en evaluar el estado de salud oral general a través de un examen clínico estomatológico (número de dientes, caries, índice COP, cálculo dental, restos radiculares, obturaciones, estado periodontal, uso, tipo y estado de prótesis dentales), utilizando luz artificial y espejo oral No. 5 y realizado por dos odontólogos previamente calibrados (Kappa >0,80).

Para el análisis estadístico se calcularon promedios, desviación estándar, distribución de frecuencias y porcentajes. Se estimó ocurrencia del estado de salud oral y niveles de percepción CVRSB mediante prevalencias, calculando intervalos de confianza al $95 \%$. Se evaluaron relaciones entre variables utilizando la prueba Chi cuadrado, con un límite de decisión de 0,05. Todos los valores de los estimadores fueron ajustados a partir del diseño muestral. La fuerza de asociación se estimó mediante Razones de Odds (OR). Para el análisis multivariable se utilizó la regresión logística nominal, teniendo en cuenta las variables que marcaron una probabilidad igual o menor al $5 \%$. Para el análisis se utilizó el programa estadístico STATA versión para Windows 10.0. 


\section{RESULTADOS}

La muestra estuvo integrada por un total de 400 adultos que asistieron a clínicas odontológicas universitarias de la ciudad de Cartagena, con edad promedio de 44,6 años (DE=13,7), el 54,7 \% estaban en etapa de adultez madura y el $66,7 \%$ eran de sexo femenino, en su mayoría eran casados con el $27,5 \%$, católicos en un $69,2 \%$ y de procedencia urbana con el $81,5 \%$, asimismo en mayor proporción pertenecían a un nivel socioeconómico bajo o muy bajo con el 86,2 $\%$, el $79,7 \%$ tenían ingresos menores a un salario mínimo legal vigente y $27,2 \%$ poseían un nivel de formación académica técnica.

Con relación al motivo de consulta el 58,5\% acudió a las clínicas odontológicas universitarias por problemas estéticos, seguido por alteraciones en la oclusión; con relación al estado de salud oral el 89,5 $\%$ presentó un índice COP superior a 3, el $59 \%$ tenía un índice de placa bacteriana superior al $15 \%$, el 50 $\%$ presentó restos radiculares y $35,7 \%$ usaban prótesis dentales desadaptadas; con relación a los tratamientos realizados $62 \%$ había recibido acciones de promoción y prevención de salud oral (Tabla I). Respecto al nivel de percepción del CVRSB según el índice GOHAI, el 60,2 \% (241 pacientes) (IC $95 \%$ : 55 65) presentó un impacto bajo de su salud oral sobre la
CV, seguido de un impacto alto con el 33,2 \% (133 pacientes) (IC $95 \%$ : 28 - 37) y la frecuencia más baja fue el impacto moderado con un 6,5\% (26 pacientes) (IC $95 \%$ : 4-8). Al evaluar la asociación entre el impacto de la salud oral con las características sociodemográficas se encontraron diferencias estadísticamente significativas entre el bajo nivel de escolaridad ( $p=0,000 ;$ OR $=2,4$; IC $95 \%=1,6-3,8)$, no presencia de seguridad social en salud $(p=0,002$; OR=1,9; IC $95 \%=1,2-2,8)$, con un alto impacto de CVRSB (Tabla II). Respecto a la asociación del impacto CVRSB con el estado de salud oral se encontraron diferencias estadísticamente significativas con la presencia de menos de 19 dientes $(p=0,000$; $\mathrm{OR}=3,6$; IC $95 \%=2,3-5,8)$, restos radiculares $(p=0,000 ; O R=6,5 ;$ IC $95 \%=4,1-10)$, cálculos $(p=0,000 ; O R=5,3 ;$ IC $95 \%=3,3-8,4)$, cuando el motivo de consulta no era por control $(p=0,004 ; O R=0,23$; IC $95 \%=0,07-0,6)$ y cuando no le han realizado tratamiento de promoción y prevención $(p=0,000 ; O R=0,29$; IC $95 \%=0,19-0,4)$; al realizar el modelo multivariado, las variables que producen un impacto negativo sobre la CVRSB fueron el no contar con servicios de seguridad social (salud), presencia de menos de 19 dientes, de restos radiculares y de cálculos dentales $(p<0,05)$ mientras que la presencia de altos índices de placa bacteriana y el pertenecer a bajos estratos socioeconómicos no presentó impacto negativo sobre la CVRSB (Tabla II).

Tabla I. Diagnóstico de salud oral de los adultos jóvenes y maduros de las Clínicas Universitarias de la Universidad de Cartagena y la Corporación Universitaria Rafael Núñez, Colombia.

\begin{tabular}{lccc}
\hline Diagnóstico de Salud Oral & Frecuencia $\mathrm{n}=400$ & Porcentajes & $\mathrm{IC}=95 \%$ \\
\hline Motivo de consulta & 41 & 10,2 & $7,2-13$ \\
Dolor & 151 & 37,7 & $32-42$ \\
Problemas en la oclusión & 234 & 58,5 & $53-63$ \\
Problemas estéticos & 28 & 7 & $4,4-9,5$ \\
Control odontológico & & & \\
Estado de Salud Oral & 105 & 26,2 & $21-30$ \\
Menos de 19 dientes & 59 & 14,7 & $11-18$ \\
Uso de prótesis dental & 20 & 35,7 & $22-48$ \\
Prótesis desajustada & 236 & 59 & $54-63$ \\
Placa bacteriana superior al $15 \%$ & 220 & 55 & $50-59$ \\
Cálculo dental & 200 & 50 & $45-54$ \\
Restos radiculares & 358 & 89,5 & $86-92$ \\
COP superior a 3 & 114 & 28,5 & $24-32$ \\
Obturaciones desajustadas & 7 & 1,75 & $0,4-3$ \\
Problemas de ATM & & & \\
Tratamientos realizados & 248 & 62 & $57-66$ \\
Promoción y prevención & 71 & 17,7 & $13-21$ \\
Cirugía & 45 & 11,2 & $8,1-14$ \\
Endodoncia & 41 & 10,2 & $7,2-13$ \\
Rehabilitación oral & 77 & 19,2 & $15-23$ \\
Operatoria Simples & & & \\
\hline
\end{tabular}


DÍAZ-CÁRDENAS, S.; MEISSER-VIDAL, M. A.; TIRADO-AMADOR, L. R.; FORTICH-MESA N.; TAPIAS-TORRADO, L. \& GONZÁLEZ-MARTÍNEZ, F. D. Impacto de salud oral sobre calidad de vida en adultos jóvenes de clínicas odontológicas universitarias. Int. J. Odontostomat., 11(1):5-11, 2017.

Tabla II. Asociación del Impacto de la salud oral sobre la calidad de vida con las características sociodemográficas y las relacionadas con el estado de salud oral de los participantes.

\begin{tabular}{|c|c|c|c|c|c|c|c|c|c|c|}
\hline \multirow{3}{*}{$\begin{array}{l}\text { Características sociodemográficas } \\
\text { Edad }\end{array}$} & \multicolumn{4}{|c|}{ Impacto de la SB sobre la CV } & \multirow{3}{*}{ Valor $p$} & \multicolumn{2}{|c|}{ Bivariado } & \multirow{3}{*}{\multicolumn{2}{|c|}{$\begin{array}{l}\text { Multivariado* } \\
\text { OR }\end{array}$}} & \multirow[b]{2}{*}{ IC $95 \%$} \\
\hline & \multicolumn{2}{|c|}{ Alto } & & & & OR & IC $95 \%$ & & & \\
\hline & $\mathrm{n}$ & $\%$ & $\mathrm{n}$ & $\%$ & & & & & & \\
\hline Adultos Jóvenes & 66 & 36,4 & 115 & 63,5 & 0,2 & & & & $\dagger$ & \\
\hline Adultos Maduros y Ma yores & 93 & 42,5 & 126 & 57,5 & & & & & & \\
\hline Sexo & & & & & & & & & & \\
\hline Masculino & 54 & 40,6 & 79 & 59,4 & 0,8 & 1,05 & $0,6-1,6$ & & $\dagger$ & \\
\hline Femenino & 105 & 39,3 & 162 & 60,7 & & & & & & \\
\hline Estado Civil & & & & & & & & & & \\
\hline Casado & 82 & 40,6 & 120 & 59,4 & 0,7 & 1,07 & $0,7-1,6$ & & $\dagger$ & \\
\hline Soltero & 77 & 38,9 & 121 & 61,1 & & & & & & \\
\hline Nivel de Escolaridad & & & & & & & & & & \\
\hline Bajo & 68 & 54,8 & 56 & 45,2 & 0,000 & 2,4 & $1,6-3,8$ & & $\dagger$ & \\
\hline Alto & 91 & 33 & 185 & 67 & & & & & & \\
\hline Estrato Socioeconómico & & & & & & & & & & \\
\hline Bajo & 149 & 38,7 & 236 & 61,3 & 0,03 & 0,3 & $0,1-0,9$ & 0,27 & $0,07-0,9$ & 0,04 \\
\hline Alto & 10 & 66,7 & 5 & 33,3 & & & & & & \\
\hline Ingresos Socioeco nómicos & & & & & & & & & & \\
\hline Menores a un SMMLV & 119 & 37,2 & 201 & 62,8 & 0,03 & 0,6 & $0,3-0,9$ & & $\dagger$ & \\
\hline Mayores a un SMMLV & 40 & 50 & 40 & 50 & & & & & & \\
\hline Seguridad Social & & & & & & & & & & \\
\hline No presenta & 92 & 47,6 & 101 & 52,3 & 0,002 & 1,9 & $1,2-2,8$ & 2,2 & $1,3-3,6$ & 0,001 \\
\hline Presenta & 67 & 32,3 & 140 & 67,3 & & & & & & \\
\hline $\begin{array}{l}\text { Variables relacionadas con el Esta } \\
\text { Salud oral }\end{array}$ & & & & & & & & & & \\
\hline Presencia de Dientes & & & & & & & & & & \\
\hline Menos de 19 dientes & 66 & 62,8 & 39 & 37,1 & 0,000 & 3,6 & $2,3-5,8$ & 1,8 & $1,07-3,1$ & 0,02 \\
\hline Más de 19 dientes & 93 & 31,5 & 202 & 68,5 & & & & & & \\
\hline Presencia de Restos Radiculares & & & & & & & & & & \\
\hline $\mathrm{Si}$ & 121 & 60 & 81 & 40 & 0,000 & 6,5 & $4,1-10$ & & & \\
\hline No & 38 & 19,2 & 160 & 80,8 & & & & 2,3 & $1,27-4,2$ & 0,006 \\
\hline Obturaciones Desadaptadas & & & & & & & & & & \\
\hline $\mathrm{Si}$ & 53 & 43,5 & 61 & 53,5 & 0,08 & 1,4 & $0,9-2,2$ & & $\dagger$ & \\
\hline No & 106 & 37 & 180 & 63 & & & & & & \\
\hline Presencia de Cálculos Dentales & & & & & & & & & & \\
\hline $\mathrm{Si}$ & 124 & 56,4 & 96 & 43,6 & 0,000 & 5,3 & $3,3-8,4$ & 2,6 & $1,5-4,4$ & 0,001 \\
\hline No & 35 & 19,4 & 145 & 80,5 & & & & & & \\
\hline Presencia de Placa Bacteriana & & & & & & & & & & \\
\hline Superior al $15 \%$ & 60 & 25,4 & 176 & 74,6 & 0,000 & 0,22 & $0,1-0,3$ & 0,48 & $0,2-0,8$ & 0,009 \\
\hline Inferior al $15 \%$ & 99 & 60,3 & 65 & 39,6 & & & & & & \\
\hline Presencia de Prótesis Desadaptada & & & & & & & & & & \\
\hline $\mathrm{Si}$ & 10 & 50 & 10 & 50 & 0,4 & 1,5 & $0,5-4,7$ & & $\dagger$ & \\
\hline No & 14 & 38,9 & 22 & 61,1 & & & & & & \\
\hline Problemas de ATM & & & & & & & & & & \\
\hline $\mathrm{Si}$ & 3 & 42,8 & 4 & 57,2 & 0,8 & 1,13 & $0,2-5,2$ & & $\dagger$ & \\
\hline No & 156 & 39,7 & 237 & 60,3 & & & & & & \\
\hline Motivos de Consulta & & & & & & & & & & \\
\hline Problemas estéticos & & & & & & & & & & \\
\hline $\mathrm{Si}$ & 97 & 41,4 & 137 & 58,5 & 0,4 & 1,18 & $0,7-1,7$ & & $\dagger$ & \\
\hline No & 62 & 37,3 & 104 & 62,6 & & & & & & \\
\hline Problemas de Oclusión & & & & & & & & & & \\
\hline $\mathrm{Si}$ & 40 & 26,5 & 111 & 73,5 & 0,000 & 0,4 & $0,2-0,6$ & & $\dagger$ & \\
\hline No & 119 & 47,8 & 130 & 52,2 & & & & & & \\
\hline Problemas de dolor & & & & & & & & & & \\
\hline $\mathrm{Si}$ & 21 & 51,2 & 20 & 48,8 & 0,1 & 1,6 & $0,8-3,2$ & & $\dagger$ & \\
\hline No & 138 & 38,4 & 221 & 61,6 & & & & & & \\
\hline Control Odontológico & & & & & & & & & & \\
\hline $\mathrm{Si}$ & 4 & 14,3 & 24 & 85,7 & 0,004 & 0,23 & $0,07-0,6$ & & $\dagger$ & \\
\hline No & 155 & 41,7 & 217 & 58,3 & & & & & & \\
\hline Tratamientos Realizados & & & & & & & & & & \\
\hline Promoción y Prevención & & & & & & & & & & \\
\hline Si & 71 & 28,6 & 177 & 71,3 & 0,000 & 0,29 & $0,1-0,4$ & & $\dagger$ & \\
\hline No & 88 & 57,9 & 64 & 42,1 & & & & & & \\
\hline Cirugía & & & & & & & & & & \\
\hline $\mathrm{Si}$ & 46 & 64,8 & 25 & 35,2 & 0,000 & 3,5 & $2-6$ & & $\dagger$ & \\
\hline No & 113 & 34,3 & 216 & 65,6 & & & & & & \\
\hline Endodoncia & & & & & & & & & & \\
\hline $\mathrm{Si}$ & 26 & 57,8 & 19 & 42,2 & 0,01 & 2,2 & $1,2-4,2$ & & $\dagger$ & \\
\hline No & 133 & 37,4 & 222 & 62,5 & & & & & & \\
\hline Rehabilitación & & & & & & & & & & \\
\hline $\mathrm{Si}$ & 19 & 46,3 & 22 & 53,6 & 0,3 & 1,3 & $0,7-2,5$ & & $\dagger$ & \\
\hline No & 140 & 39 & 219 & 61 & & & & & & \\
\hline Operatoria & & & & & & & & & & \\
\hline $\mathrm{Si}$ & 17 & 22 & 60 & 78 & 0,001 & 0,3 & $0,2-0,6$ & & $\dagger$ & \\
\hline No & 142 & 44 & 181 & 56 & & & & & & \\
\hline
\end{tabular}


DÍAZ-CÁRDENAS, S.; MEISSER-VIDAL, M. A.; TIRADO-AMADOR, L. R.; FORTICH-MESA N.; TAPIAS-TORRADO, L. \& GONZÁLEZ-MARTíNEZ, F. D. Impacto de salud oral sobre calidad de vida en adultos jóvenes de clínicas odontológicas universitarias. Int. J. Odontostomat., 11(1):5-11, 2017.

\section{DISCUSIÓN}

El impacto de la salud oral sobre la calidad de vida puede generar grandes beneficios al informar sobre aspectos tales como sentimientos y percepciones a nivel individual y colectivo, lo que favorece una comunicación efectiva entre profesionales y pacientes y quizás sirva para medir los resultados clínicos de los servicios prestados (Bennadi \& Reddy) por lo que se recomienda realizar estudios longitudinales que permitan el seguimiento a estos cambios en relación a la salud oral y calidad de vida; así mismo se sugiere utilizar estos hallazgos para el diseño e implementación de estudios de intervención que logren prevenir condiciones orales desfavorables para la calidad de vida.

Los hallazgos del presente estudio evidencian con respecto a los niveles de percepción de CVRSB, a través del índice del GOHAI, un bajo impacto de la Salud Oral sobre la Calidad de Vida en más de la mitad de los sujetos a pesar de destacarse una historia de caries dental preocupante dado que el COP estuvo por encima de 3 en la mayoría de los sujetos valorados comparando las cifras reportadas en Colombia, donde el índice de dientes COP se considera que presenta incrementos permanentes de 5 dientes afectados a medida que avanza la edad (Martínez et al., 2013). Otros hallazgos, como presencia de cálculo dental, restos radiculares y contar con menos de 19 dientes en la cavidad oral mostraron un impacto negativo sobre la CVRSB.

Es probable que los adultos jóvenes y maduros del estudio por su condición física general aventajada frente a los adultos mayores no precisen un mejor estado de salud oral para mantenerse saludables, contrario en algunos adultos mayores que presentan complicaciones en su estado de salud general y que cualquier afección como un mal estado de salud oral podría complicar su bienestar y calidad de vida. Díaz et al., valoró el impacto de la salud oral en la calidad de vida de adultos mayores, reportando presencia de prótesis desadaptadas en más de la mitad de la muestra estudiada, cálculos dentales en el $80 \%$ y de restos radiculares en menos del $30 \%$, ante lo cual se puede inferir la existencia de diferentes condiciones orales según la edad de los individuos valorados. Resulta importante considerar que la prevalencia de estos hallazgos, varía en las distintas edades de la adultez lo que hace necesario considerar la posibilidad de implementar programas que permitan hacer promoción y prevención en salud oral en esta población que a futuro representará a la población mayor, para los cuales está ampliamente documentada la existencia de trastornos orales como pérdida de dientes (Reich et al., 2011) y deterioro de la condición periodontal con subsecuentes problemas de masticación, dolor, dificultades al comer, y problemas en las relaciones sociales (Esquivel \& Jiménez, 2010). Por otro lado, este estado de salud oral hallado en el estudio no se espera encontrar en este grupo de adultos jóvenes. Esta población joven y madura corresponde a los adultos trabajadores que con escasa disponibilidad de tiempo y demandas laborales podrían dificultar la asistencia a los servicios odontológicos, cuyos horarios de oferta no resultan atractivos para este grupo y a largo plazo generaría un descuido en la salud oral de la población productiva de un país. Por ello se ameritarían programas de promoción y prevención que acudan a los espacios laborales de esta población y contribuyan a disminuir los índices de morbilidad oral que a largo plazo conllevarían a un ausentismo laboral, enfermedades sistémicas y disminución de la producción económica de un país.

Según Martins et al. (2007) las consultas odontológicas, al contrario de las consultas médicas, tienden a disminuir en la medida en que los habitantes son más viejos. Por tanto, la utilización de los servicios odontológicos aumenta durante la adultez mayor (Martins et al.), este estado de salud oral inadecuado desde edades tempranas generaría una disminución de la función diaria, actividad física y compromiso en los dominios físicos de la calidad de vida relacionada con salud (Mack et al., 2005), lo que a su vez repercute en una mayor prevalencia de la obesidad y junto a esta mayor riesgo de enfermedades cardiovasculares y trastornos gastrointestinales, aumento del riesgo de diabetes mellitus no dependiente de la insulina, aumento del riesgo de alteraciones electrocardiográficas, hipertensión, insuficiencia cardíaca, enfermedad isquémica del corazón, derrame cerebral y esclerosis válvula aórtica (Emami et al., 2013) teniendo en cuenta que estas enfermedades ya no se presentan de forma exclusiva hacia la adultez mayor, lo que podría predecir una relación entre el estado de salud oral y complicaciones de salud en adultos jóvenes y maduros y reformular políticas en salud pública que articulen el manejo de enfermedades que generen altas cifras de morbimortalidad en esta población con el seguimiento y control del estado de salud oral de los mismos que pueda complicar el 
pronóstico y tratamiento de estas enfermedades. Así, Holmlund et al. (2010), concluyó que el número de dientes era un predictor dependiente de la dosis a la mortalidad cardiovascular. Así mismo, la presencia de restos radiculares puede convertirse en un foco séptico local que podría facilitar infecciones sistémicas (Shay, 2002), ocasionar traumatismo en tejidos blandos mucosos de cavidad oral, obstaculizar el desarrollo idóneo de la alimentación e incluso pueden reprimir a los individuos en su dimensión social (relaciones interpersonales) por la existencia de una percepción de condiciones orales no saludables y sin estéti$\mathrm{ca}$, tal como se reporta en el estudio realizado por Martins et al. (2015), en el que la ausencia de restos radiculares aumenta significativamente la prevalencia de una buena percepción de salud oral, la cual también se encuentra inmersa en los ideales de salud general y por ende calidad de vida. Por otro lado, la presencia de cálculo dental juega un papel importante en la etiología y progresión de la enfermedad periodontal que a su vez puede aumentar la gravedad de los olores desagradables provenientes de la cavidad oral (halitosis), con la mayor producción de compuestos volátiles de azufre (Bolepalli et al., 2015). Lo anterior compromete la fisiología de la cavidad oral y la presencia de halitosis asociada, posiblemente puede afectar la dimensión psicosocial al generar en los individuos cohibición en la interacción con otros por vergüenza e interferir en las relaciones sociales de esta población y comprometer las tareas propias de la etapa como la consolidación de núcleos familiares y estabilidad laboral (Erikson, 1981).

Así mismo, el acceso a los servicios de salud genera tranquilidad para los individuos y poblaciones, pero muchas veces por la naturaleza de prestación de estos servicios sistemas de salud se convierte en una preocupación, tal y como sucede para el caso de Colombia quien ha adoptado un sistema regulado, con el firme propósito de mejorar el acceso a los servicios, sin embargo pese a esto aún hay algunas limitaciones en el acceso (Vargas et al., 2010). Al respecto aquí se reporta el efecto negativo de la ausencia de servicios de salud sobre la calidad de vida, pues en estos servicios se encuentran incluidos todos los niveles de atención en salud, que requieren los sujetos tanto para enfermedades generales como las enfermedades orales y desde las acciones preventivas hasta las terapéuticas, con las que se puede favorecer al bienestar integral de los mismos, coincidiendo con lo reportado por Topal et al. (2012) tras considerar los desafíos en el acceso a los servicios de salud y su efecto en la calidad de vida de una población de inmigrantes, lo que quizás empeora dicha condición de accesibilidad. Para el caso de los adultos jóvenes y maduros del estudio, más de la mitad cuenta con el mínimo de ingresos necesarios para costear gastos de la canasta familiar, lo que limitaría los recursos económicos para realizar pagos a los servicios de salud y por ende comprometer el bienestar integral y la salud oral, a pesar de contar el sistema de salud colombiano con un régimen subsidiado que da cabida para aquellos que no posean la capacidad de pago, pero aparecen otros factores como los mitos frente al uso de estos servicios vistos de baja calidad, dificultad en el acceso y pocas actividades de promoción y prevención en salud que motiven a los pacientes a acudir a los servicios odontológicos (Rocha, 2013).

En conclusión, la salud oral presenta un gran impacto en la percepción de la calidad de vida de adultos jóvenes y maduros que asisten a las clínicas universitarias de la ciudad de Cartagena, lo cual sugiere la necesidad de mantener la cavidad oral con buen estado de salud desde edades tempranas, que contribuya a la salud general y por ende al bienestar de las personas, proporcionando una mejor calidad de vida.

DÍAZ-CÁRDENAS, S.; MEISSER-VIDAL, M. A.; TIRADOAMADOR, L. R.; FORTICH-MESA N.; TAPIAS-TORRADO, L. \& GONZÁLEZ-MARTíNEZ, F. D. Oral health impact on life quality in young adults at dental university clinics. Int. J. Odontostomat., 11(1):5-11, 2017.

ABSTRACT: The aim of this study is to associate oral health status and quality of life in adults treated at university dental clinics in Cartagena, Colombia second semester 2013. Cross-sectional analytical study, in 400 young and mature adults (20-59 years) attending dental clinics University and the University Corporation Cartagena Rafael Nunez and distributed equally between the two institutions; a validated instrument was applied to investigate for sociodemographic variables and the General Oral Health Assessment Index (GOHAl) evaluating the impact of oral health on quality of life (CVRSB); oral exam was performed to evaluate the number of teeth, presence of dental caries and plaque, root fragments, dental calculus and seals. Data were analyzed by frequency and proportion, taking confidence intervals of $95 \%$, significance of relationships between variables by means of chi-square, risk estimation through OR, and logistic regression model with a confidence level of $95 \% .66 .7 \%$ of participants were women and were at the stage of mature adulthood with $54.7 \%$, with an average age of 44.6 years $(\mathrm{SD}=13.7) .60 .2 \%(95 \% \mathrm{Cl} 55-65)$ had a low impact of oral health on quality of life, followed by a high impact with 33.2 $\%$. We found associations between low levels of schooling $(p=0.000 ; O R=2.4 ;$ IC $95 \%=1.6-3.8)$, social security $(p=0.0 .02 ; O R=1.9 ;$ IC $95 \%=1.2-2.8)$, presence of less than 
19 teeth $(p=0.000 ; O R=3.6 ; \operatorname{IC~} 95 \%=2.3-5.8)$, root fragments $(p=0.000 ; O R=6.5 ;$ IC $95 \%=4.1-10)$, dental calculus $(p=0.000 ; O R=5.3 ;$ IC $95 \%=3.3-8.4)$ with the negative impact of the CVRSB. In the multivariate model variables that best explain the negative impact of CVSB they were: lack of social security, have fewer than 19 teeth, presence of root fragments and stones $(p<0.05)$. Young and mature adults have a negative impact on CVRSB especially when they have less than 19 teeth, presence of root fragments and dental calculus or when they have no social security arises.

KEY WORDS: oral health, quality of life, self perception.

\section{REFERENCIAS BIBLOGRÁFICAS}

Atchison, K. A. \& Dolan, T. A. Development of the geriatric oral health assessment index. J. Dent. Educ., 54(11):680-7, 1990.

Bennadi, D. \& Reddy, C. V. K. Oral health related quality of life. J. Int. Soc. Prev. Community Dent., 3(1):1-6, 2013.

Bolepalli, A. C.; Munireddy, C.; Peruka, S.; Polepalle, T.; Choudary Alluri, L. S. \& Mishaeel, S. Determining the association between oral malodor and periodontal disease: A case control study. J. Int. Soc. Prev. Community Dent., 5(5):413-8, 2015.

Concha Sánchez, S. C. \& Camargo Lemus, D. M. Análisis de la asociación entre la calidad vida y la condición oral de las personas mayores vinculadas a tres instituciones geriátricas de Bucaramanga: II Fase. Rev. Ustasalud Odontol., 6:75-86, 2007.

Díaz Cárdenas, S.; Arrieta Vergara, K. \& Ramos Martínez, K. Impacto de la salud oral en la calidad de vida de adultos mayores. Rev. Clin. Med. Fam., 5(1):9-16, 2012.

Emami, E.; de Souza, R. F.; Kabawat, M. \& Feine, J. S. The impact of edentulism on oral and general health. Int. J. Dent., 2013:498305, 2013.

Erikson, E. H. \& Katchadourian, H. A. La Adultez. México D. F., Fondo de Cultura Económica, 1981

Esquivel, H. R. I. \& Jiménez, F. J. Necesidades de atención odontológica en adultos mayores mediante la aplicación del GOHAl. Rev. Asoc. Dent. Mex., 67(3):127-32, 2010.

Gherunpong, S.; Tsakos, G. \& Sheiham, A. The prevalence and severity of oral impacts on daily performances in Thai primary school children. Health Qual. Life Outcomes, 2:57, 2004.

Hernández-Mejía, R.; Calderón-García, R.; Fernández-López, J. A. \& Cueto-Espinar, A. Escala para medir la calidad de vida en desdentados totales rehabilitados con prótesis completa mucosoportada. RCOE, 11(2):181-91, 2006.

Holmlund, A.; Holm, G. \& Lind, L. Number of teeth as a predictor of cardiovascular mortality in a cohort of 7,674 subjects followed for 12 years. J. Periodontol., 81(6):870-6, 2010.

Kushnir, D.; Zusman, S. P. \& Robinson, P. G. Validation of a Hebrew version of the Oral Health Impact Profile 14. J. Public Health Dent., 64(2):71-5, 2004.

Locker, D. \& Allen, F. What do measures of 'oral health-related quality of life' measure? Community Dent. Oral Epidemiol., 35(6):40111, 2007.

Mack, F.; Schwahn, C.; Feine, J. S.; Mundt, T.; Bernhardt, O.; John, U.; Kocher, P. T. \& Biffar, R. The impact of tooth loss on general health related to quality of life among elderly Pomeranians: results from the study of health in Pomerania (SHIP-O). Int. J. Prosthodont., 18(5):414-9, 2005.

Martínez-Pabón, M. C.; Morales-Uchima, S. M. \& Martínez-Delga- do, C. M. Caries dental en adultos jóvenes en relación con características microbiológicas y fisicoquímicas de la saliva. Rev. Salud Pública, 15(6):867-77, 2013.

Martins, A. M. E. B. L.; Barreto, S. M. \& Pordeus, I. A. Uso de serviços odontológicos entre idosos brasileiros. Rev. Panam. Salud Publica, 22(5):308-16, 2007.

Martins, A. B.; Dalberto, C. da S. \& Hugo, F. N. Associação entre a presença de restos radiculares e a autopercepção de saúde bucal em idosos. Ciênc. Saúde Coletiva, 20(12):3669-79, 2015.

Meller, C. Importancia de la odontología preventiva en el adulto mayor: Una aproximación personal Odontol. Prev., 1(2):73-82, 2008.

Misrachi, C. \& Espinoza, I. Utilidad de las mediciones de la calidad de vida relacionada con la salud. Rev. Dent. Chile, 96(2):28-35, 2005.

Montes S., J. L. Impacto de la Salud Oral en la Calidad de Vida del Adulto Mayor. Rev. Dent. Chile, 92(3):29-31, 2001.

Reich, K. M.; Huber, C. D.; Lippnig, W. R.; Ulm, C.; Watzek, G. \& Tangl, S. Atrophy of the residual alveolar ridge following tooth loss in an historical population. Oral Dis., 17(1):33-44, 2011.

Rocha-Buelvas, A. Análisis sobre el acceso a los servicios de la salud bucal: un indicador de equidad. Rev. Gerenc. Polit. Salud (Bogotá), 12(25):96-112, 2013.

Shay, K. Infectious complications of dental and periodontal diseases in the elderly population. Clin. Infect. Dis., 34(9):1215-23, 2002.

Topal, K.; Eser, E.; Sanberk, I.; Bayliss, E. \& Saatci, E. Challenges in access to health services and its impact on quality of life: a randomised population-based survey within Turkish speaking immigrants in London. Health Qual. Life Outcomes, 10:11, 2012.

Vargas-Lorenzo, I.; Vásquez-Navarrete, M. L. \& Mogollón-Pérez, A. $\mathrm{S}$. Acceso a la atención en salud en Colombia. Rev. Salud Pública, 12(5):701-12, 2010.

Study protocol for the World Health Organization project to develop a Quality of Life assessment instrument (WHOQOL). Qual. Life Res., 2(2):153-9, 1993.

Zini, A. \& Sgan-Cohen, H. D. The effect of oral health on quality of life in an underprivileged homebound and non-homebound elderly population in Jerusalem. J. Am. Geriatr. Soc., 56(1):99-104, 2008.

Dirección para correspondencia:

Shyrley Díaz-Cárdenas

Facultad de Odontología

Universidad de Cartagena

Cartagena de Indias

COLOMBIA

Email: sdiazc@unicartagena.edu.co

Recibido :15-02-2016

Aceptado:23-12-2016 